\title{
Risk Factors And Quality Of Life Of Adult Patients With Chronic Voice Disorders
}

\author{
E. A. El-Moselhy, Y. A. Barka, E. S. Abd-Allah*; \\ T. S. Alshorbagy**; M. M. El-Sawy** And T. M. Farghaly** \\ Departments of Community Medicine; \\ Community Health Nursing* and Oto-Rhino-Laryngology** \\ Faculty of Medicine and Nursing, Al-Azhar and Zagazig University
}

\begin{abstract}
The aim of the present research is to determine the common causes of chronic voice disorders, to determine the sociodemographic and behavioral risk factors for patients with chronic voice disorders and to study the QOL of them. The ORL Outpatient Clinics, Al-Azhar University hospitals were chosen to carry out this study. A total of 495 patients with chronic voice disorders and a control group of the same number were enrolled in the study. A casecontrol, hospital based study design was used. The most common causes of chronic voice disorders among these patients were chronic laryngitis (35.6\%), vocal fold nodules $(22.6 \%)$, functional dysphonia (18.6\%) and vocal fold polyps (13.5\%). The 25-44 years age group, low social class, sale man occupation, urban residence and female gender were the most important significant sociodemographic risk factors for patients with chronic voice disorders (ORs $=4.17$, $2.01,1.71,1.60$ and 1.32, respectively). The +ve reflux symptoms index, voice abuse and smoking were an important significant clinical risk factors (ORs=16.94, 8.33 and 6.01, respectively). Also, patients with chronic voice disorders had a significantly poorer self-reported health related domain scores than the controls on all eight $\mathrm{SF}-36$ domains $(\mathrm{P}=0.00)$. Moreover, patients with chronic voice disorders due to different laryngeal diseases had a significantly poorer self-reported health related domain scores than the controls on all eight SF-36 domains except in the miscellaneous diseases group.
\end{abstract}

\section{Introduction}

Voice is one of the unique attributes of humans. It provides a principal means of communication, emotional expression and identity (Solomon et al., 2003). Voice disorders exist when quality, pitch or loudness differs from others of the same age, gender, cultural background and geographic location, thereby drawing attention to the speaker. Voice disorders may results from changes in the structure and/or function of the laryngeal mechanism (Stemple et al., 1996). Laryngeal pathologies that cause voice disorders comprise a group of diseases; the most frequent are chronic laryngitis, nodules, polyps, edema, functional dysphonia ...etc (Herrington-Hall et al., 1988 and Coyle et al., 2001). Epidemiological reports on the occurrence of chronic voice disorders have been few in number. Also, reports are relatively scarce, outdated and provide conflicting information (Miller and Verdolini, 1995; Smith et al., 1997 and Titze et al., 2007). An update of such data may lead to further identification of subjects at risk for developing chronic voice disorders, information to enhance public education about voice disorders and identifying of risk factors associated with various demographic and clinical variables (Coyle et al., 2001 and Titze et al., 2007).

Moreover, over the past 20 years there has been an increased recognition of the patient's point of view as an important component in the assessment of health care outcomes (Watson et al., 1996). Quality of life (QOL) has become accepted as an end point in clinical research trials, as interest in patients' experiences and preferences has grown (Patrick and Bergner, 1990). Reports 
of QOL end points remain uncommon and quality of reporting is often poor (Sanders et al., 1998). Also, the term QOL is often used vaguely and without clear definition (Fallowfield, 1996). The most accepted definition of QOL is "a measure of the optimum energy or force that endows a person with the power to cope successfully with the full range of challenges encountered in the real world". The term QOL applies to all individuals, regardless of illness or handicap, on the job, at home or in leisure activities (Gotay et al., 1992). The perception of QOL varies between individuals and it is dynamic within them. QOL in relation to health is the gap between our expectations and experience of health. People with different expectations report a different QOL even when the same clinical condition is present. Current measures for QOL do not account for expectations of health (Guillemin et al., 1993 and Carr et al., 2006). There are many instruments that have been proposed to measure QOL; generic measures as the sickness impact profile (Bergner et al., 1981). Other methods include measures focusing on a single aspect such as pain or anxiety and individualized measures, in which patients define and rate the most important aspects of their QOL (Begg et al., 1996).

People with dysphonia seem to experience employment, lifestyle and social difficulties as a direct consequence of their voice disorders. Assessments of the impact of dysphonia on the patient have focused on psychological and voice outcomes. Any attempts to quantify the effects of the disorder on general health and quality of life have relied upon open-ended patient reports (Scott et al., 1997). There have been few studies of the QOL of patients with dysphonia (Benninger et al., 1998 and Spector et al., 2001).

The aim of the present research is to determine the most common causes of voice disorders, to define the sociodemographic, behavioral and clinical risk factors for patients with chronic voice disorders and to study the QOL of these patients with chronic voice disorders due to laryngeal diseases.

\section{Subjects And Methods}

This study was carried out in the OtoRhino-Laryngelogy (ORL) Out-patient Clinics, Al-Azhar University Hospitals in Cairo and Assiut. A total number of 495 patients with chronic voice disorders due to laryngeal diseases and an equal number of adult controls were enrolled in this study. The control group was chosen randomly from adults attending the clinics for reasons other than chronic voice disorders and found to be free. A case-control, hospital based study design was chosen to carry out this study. Chronic was defined as a period $\geq 4$ weeks. The chronic voice disorder patients and controls were adults, their age was $\geq 18$ years. The purpose of the study was explained to the patients and controls. A verbal consent of both of them, to participate in the study, was given.

Clinical examinations had been done for the patient and control groups. Also, the required investigations had been done for the patients. Laryngeal diseases that caused chronic voice disorders were diagnosed through specific protocol according to ElMoselhy et al. (2004). Also, a comprehensive questionnaire was designed to contain data relevant to the topic of the study.

The reflux symptoms index (RSI) was used to determine presence of reflux symptoms. Normative data suggests that a RSI of $\geq 10$ is clinically significant. Subjects were classified as having +ve RSI, if scored $\geq 10$ and $-v e$ RSI, if scored $<10$. Presence of +ve or -ve RSI was related to presence or absence of laryngeal pathologies (Belafsky et al., 2002).

We used the medical outcomes study 36-item short form (SF-36) to study the QOL of the patients with chronic voice disorders due to laryngeal diseases. SF-36 is one of the most widely accepted, used and psychometrically sound instrument designed to measure general health items (Watson et al., 1996). The reliability and validity of the SF-36 have been documented. Also, health functioning changed in the hypothesized direction with increased age, socioeconomic status and disease status in a population-based study suggested that the instrument is sensitive to 


\section{E. A. El-Moselhy et al}

changes in the health of the general population (Garratt, 2002). It allows investigators to explore the interaction and relative effect of multiple health conditions in the same patient. The SF-36 is containing 36 questions; each patient is scored from 0 (worst) to 100 (best) on 8 separate domains of health-related QOL. These domains include physical functioning (PF), physical limitation (PL), bodily pain (BP), general health $(\mathrm{GH})$, vitality (VT), social functioning (SF), role functioning-emotional (RE) and mental health $(\mathrm{MH})$. The questionnaire is scored according to published algorithms and it takes about 10 minutes to be complete (Hemingway et al., 1997). These scales are ordered according to the degree to which they measure physical versus mental health. The 36-item question is distributed over the 8-health domains. The SF-36 quantifies a broad range of health issues and is thus acceptable for an exploratory study on QOL in conditions that may be anticipated to affect patients in a variety of ways (Ware et al., 1993). Normative data are the key to determining whether a group or an individual scores below or above the average for their country, age or sex (Fitzpatrick et al., 2001). The chronic form of the SF-36 was used to study the impact of chronic voice disorders due to laryngeal diseases on QOL. Impact of chronic voice disorder was compared with control group, and then different etiological pathologies were compared separately with the control group.

Chi-square $\left(\chi^{2}\right)$, t-test and odds ratio (OR) were used as tests of significance. The significance level for $\chi^{2}$ and $t$ was accepted if P-value $\leq 0.05$ while, OR was weighted according to value of the $95 \%$ confidence interval (CI) or exact confidence limits (ECL).

\section{Results}

Table (1) shows the frequency distribution of chronic voice disorder patients according to the etiology. It is clear that chronic laryngitis was the most common cause $(35.6 \%)$ of chronic voice disorders. Vocal fold nodules (22.6\%), functional dysphonia (18.6\%), vocal fold polyps (13.5\%) and a group of miscellaneous conditions $(9.7 \%)$ were the other etiological causes of chronic voice disorders.

Table (2) details the distribution of chronic voice disorder patients and control group according to their sociodemographic risk factors. As regard sex, female sex was found to be a significant risk factor for a subject to be a patient with chronic voice disorders; females were $56.2 \%(\mathrm{OR}=1.32$, 95\% CI: 1.02-1.71). Also, 25-44 year age group was found to be a significant risk factor for a subject to be a patient with chronic voice disorders; the patients in this group were $44.8 \% \quad(\mathrm{OR}=1.33,95 \% \mathrm{CI}$ : 1.02-1.73). At the same time, some occupations were found to be significant risk factors for chronic voice disorders. The ORs for house wife's, factory workers, teachers, sale men and retirees were 1.38, 95\% CI: $1.01-1.89 ; 1.46,95 \%$ CI: $1.01-$ $2.09 ; 1.51,95 \%$ CI: $1.01-2.27 ; 1.71,95 \%$ CI: $1.02-2.86$ and $1.69,95 \% \mathrm{CI}: 1.08-2.65$; respectively. Moreover, low social class was a significant risk factor for chronic voice disorders ( $\mathrm{OR}=2.01,95 \% \mathrm{CI}$ : 1.542.62). Lastly, urban residence was a significant risk factor for chronic voice disorders (OR=1.60, 95\% CI: 1.04-2.47).

Table (3) sssshows the distribution of chronic voice disorder patients and control group according to behavioral risk factors. As regard voice abuse, it was found to be a significant risk factor for a subject to be a patient with chronic voice disorders (OR=8.33, 95\% CI: 5.97-11.64). Moreover, smoking was a significant risk factor for chronic voice disorders $(\mathrm{OR}=6.01,95 \% \mathrm{CI}$ : 2.29-3.95). Also, alcohol intake was a significant risk factor for a subject to be a patient with chronic voice disorders (OR=2.90, 95\% ECL: 1.08-9.05).

Table (4) clears the distribution of chronic voice disorder patients and control group according to reflux symptom index risk factor. RSI was found to be a significant risk factor for a subject to be a patient with chronic voice disorders (OR=16.94, 95\% CI: 11.80-24.38).

Table (5) demonstrates the distribution of chronic voice disorder patients according to their sociodemo-graphic risk factors. As respect sex, females were found to be significantly more common among all groups of the patients with chronic voice 
disorders except the miscellaneous diseases group. Females were 55.1\%, 57.1\%, 65.2\%, $52.2 \%$ and $45.8 \%$ in the chronic laryngitis, vocal fold nodules, functional dysphonia, vocal fold polyps and miscellaneous diseases groups; respectively. Also, 25-44 year age group was found to be the most common age to represent all patients with chronic voice disorders; the chronic laryngitis $(38.6 \%)$, vocal fold nodules $(68.8 \%)$, functional dysphonia $(42.4 \%)$ and vocal fold polyps (43.3\%). While, the patients in miscellaneous diseases group were more present in the 45-64 year age group, $54.2 \%$. Moreover, the house wife and factory worker were the most common occupations found among patients with chronic voice disorders. The house wife's were more common in groups of vocal fold nodules $(29.5 \%)$, functional dysphonia $(32.6 \%)$ and vocal fold polyps (31.3\%). While, factory workers were more common in groups of chronic laryngitis $(26.7 \%)$ and vocal fold polyps $(31.3 \%)$. Also, the patients of miscellaneous diseases group were more common in the retired group $(70.8 \%)$. As regard social class, low social class was found to be non-significantly more common among all groups of the patients with chronic voice disorders except the miscellaneous diseases group. Lastly, all groups of patients with chronic voice disorders had urban residence with a statistically significant difference; the vocal fold nodules $(97.3 \%)$, functional dysphonia (94.6\%), vocal fold polyps $(91.1 \%)$, chronic laryngitis (88.6\%) and miscellaneous diseases group (83.3\%).

Table (6) illustrates the mean and standard deviation of chronic voice disorder patients and control group according to SF36 QOL domain scores. All the means and standard deviations of the eight domain scores of the SF-36 QOL of chronic voice disorder patients were less than that of the controls with statistically significant differences $(\mathrm{P}=0.000)$.

Table (7) shows the means and standard deviations of different groups of chronic voice disorder patients and control group according to the SF-36 QOL domain scores. As respect chronic laryngitis, vocal fold nodules, functional dysphonia and vocal fold polyps; the means and standard deviations of the eight domain scores of SF36 QOL were less than that of the controls with statistically significant differences. As regard the miscellaneous diseases group, the means and standard deviations of the eight domain scores of the SF-36 QOL were less than that of the controls with statistically significant differences except that of bodily pain $(\mathrm{P}=0.09)$. Patients with functional dysphonia scored the lowest scores in all of the eight domains of the SF36 QOL except physical functioning. On the other hand, patients with vocal fold nodules scored the highest scores in all of the eight domains of the SF-36 QOL except social functioning. But, patients with chronic laryngitis scored the lowest score in physical functioning domain of the SF-36 QOL.

Table (1): Frequency distribution of chronic voice disorder patients according to the etiology.

\begin{tabular}{|l|c|c|}
\hline \multirow{2}{*}{\multicolumn{1}{c|}{ Etiology }} & \multicolumn{2}{c|}{$\begin{array}{c}\text { Chronic voice disorder } \\
\text { patients (n=495) }\end{array}$} \\
\cline { 2 - 3 } & NO. & Percent \\
\hline Chronic laryngitis & 176 & 35.6 \\
Vocal fold nodule & 112 & 22.6 \\
Functional dysphonia & 92 & 18.6 \\
Vocal fold polyp & 67 & 13.5 \\
Miscellaneous: & 48 & 9.7 \\
Larynoscleroma & 13 & 2.6 \\
Vocal fold paralysis & 12 & 2.4 \\
Bowed vocal fold & 10 & 2.0 \\
Reinke's edema & 9 & 1.8 \\
Neurogenic dysphonia & 4 & 0.8 \\
\hline
\end{tabular}


Table (2): Distribution of chronic voice disorder patients and control group according to sociodemographic risk factors.

\begin{tabular}{|c|c|c|c|c|c|}
\hline \multirow[t]{2}{*}{ Variable } & \multicolumn{2}{|c|}{$\begin{array}{c}\text { Chronic } \\
\text { voice } \\
\text { disorder } \\
(n=495)\end{array}$} & \multicolumn{2}{|c|}{$\begin{array}{c}\text { Control } \\
\text { group } \\
(\mathrm{n}=495)\end{array}$} & \multirow[t]{2}{*}{ OR $(95 \%$ CI $)$} \\
\hline & NO. & $\%$ & NO. & $\%$ & \\
\hline Sex: & & & & & \\
\hline Female & 278 & 56.2 & 244 & 49.3 & $1.32(1.02-1.71)$ \\
\hline Male & 217 & 43.8 & 251 & 50.7 & $0.76(0.59-0.98)$ \\
\hline $\begin{array}{c}\text { Age (years): } \\
18-24\end{array}$ & 89 & 18.0 & 108 & 21.8 & $0.79(0.57-1.09)$ \\
\hline $25-44$ & 205 & 41.4 & 172 & 34.8 & $1.33(1.02-1.73)$ \\
\hline $45-64$ & 174 & 35.1 & 167 & 33.7 & $1.06(0.81-1.40)$ \\
\hline$\geq 65$ & 27 & 5.5 & 48 & 9.7 & $0.54(0.32-0.90)$ \\
\hline Occupation: & & & & & \\
\hline House wife & 122 & 24.6 & 95 & 19.2 & $1.38(1.01-189)$ \\
\hline Retired & 61 & 12.3 & 38 & 7.7 & $1.69(1.08-2.65)$ \\
\hline Factory worker & 98 & 19.8 & 59 & 11.9 & 1. $46(1.01-2.09)$ \\
\hline Sale man & 46 & 9.3 & 28 & 5.7 & $1.71(1.02-2.86)$ \\
\hline Teacher & 72 & 14.6 & 50 & 10.1 & $1.51(1.01-2.27)$ \\
\hline Unemployed & 29 & 5.9 & 71 & 14.3 & $0.37(0.23-0.60)$ \\
\hline Driver & 24 & 4.8 & 17 & 3.4 & $1.43(0.73-2.83)$ \\
\hline Other & 43 & 8.7 & 137 & 27.7 & $0.25(0.33-0.56)$ \\
\hline Social class: & & & & & \\
\hline Low & 247 & 49.9 & 164 & 33.1 & $2.01(1.54-2.62)$ \\
\hline Middle & 198 & 40.0 & 249 & 50.3 & $0.66(0.51-0.85)$ \\
\hline High & 50 & 10.1 & 82 & 16.6 & $0.57(0.38-0.84)$ \\
\hline Residence: & & & & & \\
\hline Urban & 453 & 91.5 & 431 & 87.1 & $1.60(1.04-2.47)$ \\
\hline Rural & 42 & 8.5 & 64 & 12.9 & $0.62(0.41-0.96)$ \\
\hline
\end{tabular}

Table (3): Distribution of chronic voice disorder patients and control group according to behavioral risk factors.

\begin{tabular}{|c|c|c|c|c|c|}
\hline \multirow{2}{*}{ Variable } & \multicolumn{2}{|c|}{$\begin{array}{c}\text { Chronic voice } \\
\text { disorder (n=495) }\end{array}$} & \multicolumn{2}{c|}{$\begin{array}{c}\text { Control group } \\
\text { (n=495) }\end{array}$} & \multirow{2}{*}{$\begin{array}{c}\text { OR (95\% CI) } \\
\text { OR (95\% ECL)* }\end{array}$} \\
\cline { 2 - 5 } & NO. & \% & NO. & \% & \\
\hline $\begin{array}{c}\text { Voice abuse: } \\
\text { Yes }\end{array}$ & 267 & 53.9 & 61 & 12.3 & $8.33(5.97-11.64)$ \\
\hline $\begin{array}{c}\text { Smoking: } \\
\text { Yes }\end{array}$ & 271 & 54.8 & 142 & 28.7 & $6.01(2.29-3.95)$ \\
\hline $\begin{array}{c}\text { Alcohol intake: } \\
\text { Yes }\end{array}$ & 17 & 3.4 & 9 & 1.8 & $2.90(1.08-9.05)^{*}$ \\
\hline
\end{tabular}

Table (4): Distribution of chronic voice disorder patients and control group according reflux symptoms index (RSI) risk factor.

\begin{tabular}{|c|c|c|c|c|c|}
\hline $\begin{array}{c}\text { Reflux } \\
\text { symptoms } \\
\text { index }\end{array}$ & \multicolumn{2}{|c|}{$\begin{array}{c}\text { Chronic voice } \\
\text { disorder }\end{array}$} & \multicolumn{2}{|c|}{$\begin{array}{c}\text { Control group } \\
(\mathbf{n}=\mathbf{4 9 5})\end{array}$} & \multirow{2}{*}{ OR (95\% CI) } \\
\cline { 2 - 5 } & NO. & \% & NO. & \% & \\
\hline +ve RSI & 322 & 65.1 & 49 & 9.9 & \\
-ve RSI & 173 & 34.9 & 446 & 90.1 & $16.94(11.80-24.38)$ \\
\hline
\end{tabular}


Table (5): Distribution of chronic voice disorder patients due to different chronic laryngeal diseases according to their sociodemographic risk factors.

\begin{tabular}{|c|c|c|c|c|c|c|c|c|c|c|c|c|}
\hline \multirow[t]{2}{*}{ Variable } & \multicolumn{2}{|c|}{$\begin{array}{c}\text { Chronic } \\
\text { laryngitis } \\
(\mathbf{n}=176)\end{array}$} & \multicolumn{2}{|c|}{$\begin{array}{l}\text { Vocal fold } \\
\text { nodule } \\
(n=112)\end{array}$} & \multicolumn{2}{|c|}{$\begin{array}{c}\text { Functional } \\
\text { dysphonia } \\
(n=92)\end{array}$} & \multicolumn{2}{|c|}{$\begin{array}{l}\text { Vocal fold } \\
\text { Polyp } \\
(n=67)\end{array}$} & \multicolumn{2}{|c|}{$\begin{array}{c}\text { Miscellaneous } \\
\text { conditions } \\
(n=48)\end{array}$} & \multirow{2}{*}{$\chi^{2}$} & \multirow{2}{*}{$\begin{array}{c}\text { P- } \\
\text { value }\end{array}$} \\
\hline & NO. & $\%$ & NO. & $\%$ & NO. & $\%$ & NO. & $\%$ & NO. & $\%$ & & \\
\hline $\begin{array}{c}\text { Sex: } \\
\text { Male } \\
\text { Female }\end{array}$ & $\begin{array}{l}79 \\
97 \\
\end{array}$ & $\begin{array}{l}44.9 \\
55.1 \\
\end{array}$ & $\begin{array}{l}48 \\
64 \\
\end{array}$ & $\begin{array}{l}42.9 \\
57.1 \\
\end{array}$ & $\begin{array}{l}32 \\
60 \\
\end{array}$ & $\begin{array}{l}34.8 \\
65.2 \\
\end{array}$ & $\begin{array}{l}32 \\
35 \\
\end{array}$ & $\begin{array}{l}47.8 \\
52.2 \\
\end{array}$ & $\begin{array}{l}26 \\
22 \\
\end{array}$ & $\begin{array}{l}54.2 \\
45.8 \\
\end{array}$ & 12.95 & 0.011 \\
\hline $\begin{array}{c}\text { Age (years): } \\
18-24 \\
25-44 \\
45-64 \\
\geq 65\end{array}$ & $\begin{array}{c}36 \\
68 \\
64 \\
8\end{array}$ & $\begin{array}{c}20.5 \\
38.6 \\
36.4 \\
4.5\end{array}$ & $\begin{array}{c}25 \\
77 \\
9 \\
1\end{array}$ & $\begin{array}{c}22.3 \\
68.8 \\
8.0 \\
0.9\end{array}$ & $\begin{array}{c}16 \\
39 \\
34 \\
3\end{array}$ & $\begin{array}{c}17.4 \\
42.4 \\
37.0 \\
3.2 \\
\end{array}$ & $\begin{array}{c}11 \\
29 \\
23 \\
4\end{array}$ & $\begin{array}{c}16.4 \\
43.3 \\
34.3 \\
6.0\end{array}$ & $\begin{array}{c}0 \\
5 \\
26 \\
17\end{array}$ & $\begin{array}{c}0.0 \\
11.4 \\
54.2 \\
35.4\end{array}$ & $\begin{array}{c}12.92 \\
51.974 \\
3.4472 . \\
80\end{array}$ & $\begin{array}{c}0.0160 . \\
000 \\
0.0000 . \\
000\end{array}$ \\
\hline $\begin{array}{c}\text { Occupation: } \\
\text { House wife } \\
\text { Retired } \\
\text { Factory worker } \\
\text { Sale man } \\
\text { Teacher } \\
\text { Unemployed } \\
\text { Driver } \\
\text { Other } \\
\end{array}$ & $\begin{array}{c}34 \\
6 \\
47 \\
26 \\
22 \\
13 \\
12 \\
16 \\
\end{array}$ & $\begin{array}{c}19.3 \\
3.4 \\
26.7 \\
14.8 \\
12.5 \\
7.4 \\
6.8 \\
9.1 \\
\end{array}$ & $\begin{array}{c}33 \\
9 \\
19 \\
8 \\
23 \\
6 \\
5 \\
9 \\
\end{array}$ & $\begin{array}{c}29.5 \\
8.0 \\
17.0 \\
7.1 \\
20.5 \\
5.4 \\
4.5 \\
8.0 \\
\end{array}$ & $\begin{array}{c}30 \\
7 \\
9 \\
8 \\
22 \\
6 \\
4 \\
6 \\
\end{array}$ & $\begin{array}{c}32.6 \\
7.6 \\
9.8 \\
8.7 \\
23.9 \\
6.5 \\
4.4 \\
6.5 \\
\end{array}$ & $\begin{array}{c}21 \\
5 \\
21 \\
3 \\
5 \\
3 \\
3 \\
6 \\
\end{array}$ & $\begin{array}{c}31.3 \\
7.5 \\
31.3 \\
4.5 \\
7.5 \\
4.5 \\
4.5 \\
8.9 \\
\end{array}$ & $\begin{array}{c}4 \\
34 \\
2 \\
1 \\
0 \\
1 \\
0 \\
6 \\
\end{array}$ & $\begin{array}{c}8.3 \\
70.8 \\
4.2 \\
2.1 \\
0.0 \\
2.1 \\
0.0 \\
12.5 \\
\end{array}$ & $\begin{array}{c}15.73 \\
170.3 \\
24.681 \\
1.73 \\
21.20 \\
2.34 \\
4.03 \\
1.53 \\
\end{array}$ & $\begin{array}{c}0.003 \\
0.0000 \text {. } \\
000 \\
0.0190 \text {. } \\
000 \\
0.673 \\
0.401 \\
0.822 \\
\end{array}$ \\
\hline $\begin{array}{l}\text { Social class: } \\
\text { Low } \\
\text { Middle } \\
\text { High }\end{array}$ & $\begin{array}{l}81 \\
73 \\
22\end{array}$ & $\begin{array}{l}46.0 \\
41.5 \\
12.5\end{array}$ & $\begin{array}{l}62 \\
38 \\
12\end{array}$ & $\begin{array}{l}55.4 \\
33.9 \\
10.7\end{array}$ & $\begin{array}{c}46 \\
39 \\
7\end{array}$ & $\begin{array}{c}50.0 \\
42.4 \\
7.6\end{array}$ & $\begin{array}{c}37 \\
24 \\
6\end{array}$ & $\begin{array}{c}55.2 \\
35.8 \\
9.0\end{array}$ & $\begin{array}{c}21 \\
24 \\
3\end{array}$ & $\begin{array}{c}43.8 \\
50.0 \\
6.2\end{array}$ & $\begin{array}{l}3.88 \\
4.59 \\
1.67\end{array}$ & $\begin{array}{l}0.422 \\
0.332 \\
0.614\end{array}$ \\
\hline $\begin{array}{l}\text { Residence: } \\
\text { Urban } \\
\text { Rural } \\
\end{array}$ & $\begin{array}{c}156 \\
20 \\
\end{array}$ & $\begin{array}{l}88.6 \\
11.4 \\
\end{array}$ & $\begin{array}{c}109 \\
3 \\
\end{array}$ & $\begin{array}{c}97.3 \\
2.7 \\
\end{array}$ & $\begin{array}{c}87 \\
5 \\
\end{array}$ & $\begin{array}{c}94.6 \\
5.4 \\
\end{array}$ & $\begin{array}{c}61 \\
6 \\
\end{array}$ & $\begin{array}{c}91.1 \\
8.9 \\
\end{array}$ & $\begin{array}{c}40 \\
8 \\
\end{array}$ & $\begin{array}{l}83.3 \\
16.7 \\
\end{array}$ & 12.00 & 0.017 \\
\hline
\end{tabular}

Table (6): Mean and standard deviation of the chronic voice disorder patients and control group according to short form-36 QOL domain score.

\begin{tabular}{|c|c|c|c|c|}
\hline SF-36 domain @score & $\begin{array}{c}\text { Patients } \\
\text { mean } \pm \text { SD }\end{array}$ & $\begin{array}{c}\text { Controls } \\
\text { mean } \pm \text { SD }\end{array}$ & $\mathbf{t}$ & P-value \\
\hline Physical functioning & $64.48 \pm 15.48$ & $83.91 \pm 12.47$ & 21.747 & 0.000 \\
\hline Physical limitation & $57.38 \pm 13.75$ & $84.76 \pm 12.51$ & 32.770 & 0.000 \\
\hline Bodily Pain & $63.77 \pm 15.28$ & $77.79 \pm 13.75$ & 15.175 & 0.000 \\
\hline General health & $59.80 \pm 14.88$ & $74.98 \pm 15.79$ & 15.566 & 0.000 \\
\hline Vitality & $53.71 \pm 12.37$ & $70.81 \pm 15.56$ & 19.139 & 0.000 \\
\hline Social functioning & $72.73 \pm 14.76$ & $87.53 \pm 11.38$ & 17.667 & 0.000 \\
\hline Emotional limitation & $65.05 \pm 16.38$ & $85.80 \pm 13.87$ & 21.509 & 0.000 \\
\hline Mental health & $65.44 \pm 14.80$ & $76.43 \pm 16.46$ & 11.033 & 0.000 \\
\hline
\end{tabular}


Table (7): Mean and standard deviation of different causes of chronic voice disorder patients and control group according to short form-36 QOL domain score.

\begin{tabular}{|c|c|c|c|c|c|c|}
\hline $\begin{array}{c}\text { SF-36 domain } \\
\text { score }\end{array}$ & $\begin{array}{c}\text { Ch Laryng. } \\
\text { Mean } \pm \text { SD } \\
\text { P-value }\end{array}$ & $\begin{array}{c}\text { VFN } \\
\text { Mean } \pm \text { SD } \\
\text { P-value }\end{array}$ & $\begin{array}{c}\text { FD } \\
\text { Mean } \pm \text { SD } \\
\text { P-value }\end{array}$ & $\begin{array}{c}\text { VFP } \\
\text { Mean } \pm \text { SD } \\
\text { P-value }\end{array}$ & $\begin{array}{c}\text { Miscellan. } \\
\text { Mean } \pm \text { SD } \\
\text { P-value }\end{array}$ & $\begin{array}{c}\text { Controls } \\
\text { Mean } \pm \text { SD }\end{array}$ \\
\hline $\begin{array}{c}\text { Physical } \\
\text { functioning }\end{array}$ & $\begin{array}{c}60.54 \pm 15.37 \\
P=0.000\end{array}$ & $\begin{array}{c}72.11 \pm 14.34 \\
P=0.000\end{array}$ & $\begin{array}{c}61.92 \pm 13.87 \\
P=0.000\end{array}$ & $\begin{array}{c}66.57 \pm 13.19 \\
P=0.000\end{array}$ & $\begin{array}{c}64.17 \pm 16.32 \\
P=0.000\end{array}$ & $83.91 \pm 12.47$ \\
\hline $\begin{array}{l}\text { Physical } \\
\text { limitation }\end{array}$ & $\begin{array}{c}53.31 \pm 10.52 \\
P=0.000\end{array}$ & $\begin{array}{c}65.82 \pm 16.68 \\
P=0.000\end{array}$ & $\begin{array}{c}51.57 \pm 8.75 \\
P=0.000\end{array}$ & $\begin{array}{c}62.47 \pm 11.98 \\
\mathrm{P}=0.000\end{array}$ & $\begin{array}{c}55.75 \pm 9.78 \\
P=0.000\end{array}$ & $84.76 \pm 12.51$ \\
\hline Bodily Pain & $\begin{array}{c}61.03 \pm 14.58 \\
P=0.000\end{array}$ & $\begin{array}{c}69.17 \pm 13.25 \\
P=0.000\end{array}$ & $\begin{array}{c}57.36 \pm 12.91 \\
P=0.000\end{array}$ & $\begin{array}{c}67.75 \pm 14.69 \\
P=0.000\end{array}$ & $\begin{array}{c}73.97 \pm 14.82 \\
P=0.092\end{array}$ & $77.79 \pm 13.75$ \\
\hline General health & $\begin{array}{c}56.91 \pm 14.19 \\
P=0.000\end{array}$ & $\begin{array}{c}64.81 \pm 15.56 \\
P=0.000\end{array}$ & $\begin{array}{c}54.97 \pm 12.18 \\
P=0.000\end{array}$ & $\begin{array}{c}65.41 \pm 14.18 \\
P=0.000\end{array}$ & $\begin{array}{c}58.95 \pm 11.43 \\
P=0.000\end{array}$ & $74.98 \pm 15.79$ \\
\hline Vitality & $\begin{array}{c}52.82 \pm 13.38 \\
P=0.000\end{array}$ & $\begin{array}{c}53.74 \pm 12.81 \\
\mathrm{P}=0.000\end{array}$ & $\begin{array}{c}51.39 \pm 8.24 \\
P=0.000\end{array}$ & $\begin{array}{c}53.25 \pm 9.78 \\
P=0.000\end{array}$ & $\begin{array}{c}52.84 \pm 9.94 \\
P=0.000\end{array}$ & $70.81 \pm 15.56$ \\
\hline $\begin{array}{c}\text { Social } \\
\text { functioning }\end{array}$ & $\begin{array}{c}73.37 \pm 14.35 \\
P=0.000\end{array}$ & $\begin{array}{c}74.03 \pm 14.88 \\
P=0.000\end{array}$ & $\begin{array}{c}65.96 \pm 12.52 \\
P=0.000\end{array}$ & $\begin{array}{c}74.85 \pm 10.66 \\
P=0.000\end{array}$ & $\begin{array}{c}68.72 \pm 11.32 \\
P=0.000\end{array}$ & $87.53 \pm 11.38$ \\
\hline $\begin{array}{l}\text { Emotional } \\
\text { limitation }\end{array}$ & $\begin{array}{c}62.71 \pm 17.54 \\
P=0.000\end{array}$ & $\begin{array}{c}71.91 \pm 13.82 \\
P=0.000\end{array}$ & $\begin{array}{c}59.13 \pm 13.81 \\
P=0.000\end{array}$ & $\begin{array}{c}70.05 \pm 12.29 \\
P=0.000\end{array}$ & $\begin{array}{c}62.43 \pm 12.69 \\
P=0.000\end{array}$ & $85.80 \pm 13.87$ \\
\hline Mental health & $\begin{array}{c}61.73 \pm 14.46 \\
P=0.000\end{array}$ & $\begin{array}{c}71.71 \pm 12.90 \\
P=0.001\end{array}$ & $\begin{array}{c}60.32 \pm 12.68 \\
P=0.000\end{array}$ & $\begin{array}{c}69.72 \pm 12.16 \\
P=0.000\end{array}$ & $\begin{array}{c}62.95 \pm 12.97 \\
P=0.000\end{array}$ & $76.43 \pm 16.46$ \\
\hline
\end{tabular}

\section{Discussion}

Voice is a unique attributes of humans; provides a principal means of communication, emotional expression and identity (Solomon et al., 2003). Also, the field of laryngology is dynamic and ever changing; at the crossroads is the larynx, a barometer of our physical and mental health (Aronson, 1980).

Spiegel et al. (2000) stated that laryngitis is the most common laryngeal diseases. While, Herrington-Hall et al. (1988) and Coyle et al. (2001) cleared that the most common diseases that cause dysphonia were vocal fold nodules, functional dysphonia, vocal fold polyps and vocal fold paralysis. This may be explained partially by the geographical and sociocultural differences between the two societies. The most obvious difference between our results and similar studies of Herrington-Hall et al. (1988) and Coyle et al. (2001) is the presence $2.6 \%$ of cases having laryngoscleroma, which it is documented to be endemic in Egypt (AbouSeif et al., 1991 and Thompson, 2002).

The female gender (table 2) was found to be a risk factor for patients with chronic voice disorders $(\mathrm{OR}=1.32,95 \% \mathrm{CI}$ : 1.02-1.71). Cooper (1973) and HerringtonHall et al. (1988) agreed with our result while, Fitz-Hugh et al. (1958) disagreed. This may be explained by the changes in the socio-cultural aspects of females across time. At the same time, Herrington-Hall et al. (1988) reported that laryngeal pathologies occurred primarily in the old age groups, $57 \%$ of their cases were over 45 years old and $22.4 \%$ over age 64. Also, Coyle et al. (2001) showed that laryngeal pathologies occurred most frequent (38.9\%) in 45-64 years age group, patients in the age groups 25-44 years and >64 years were $26.4 \%$ and $26.8 \%$ of subjects, respectively. So, our results disagree with these studies regarding age. In our study, there is tendency for patients with chronic voice disorders due to laryngeal diseases to occur in the middle age group (25-44 years) compared to the oldest age category commonly affected in Herrington-Hall et al. (1988) and Coyle et al. (2001) studies. This disagreement may have attributed to the high life expectancy in Americans, public awareness and better health education about 
the association of chronic voice problems in western countries may have influences more adults to find out the cause of dysphonia. However, Herrington-Hall et al. (1988) reported that the peak years for abuse lesions in females were 20-50. Also, Holinger and Johnston (1951) stated that of significance too was the number of women with small children that they felt required constant disciplining. Likewise, Cooper (1973) found that the most common occupation was that of homemaker. Also, Herrington-Hall et al. (1988) stated that it seems logic to agree that some occupations carry opportunities for voice abuse, as well as emotional conflicts that were presumed to underlie psychogenic disorder. They added that the peak period for abuse lesions in females was coinciding with the childerearing years. Also, they suggested that working conditions that require speaking over noise and breathing irritants in the air, as well as physical exertion are possible causes of voice disorders in this population. In addition, Cleary (1982) and Hibbard \& Pop (1986) cleared that women's number was increased in the labor force and consequently more exposure to occupational hazards, increase numbers of cigarette smoking among them, in addition to their responsibilities as primary caretakers of home and family. Also, women appear to be more interested in health, more likely to recognize bodily changes as symptoms of an illness and more apt to seek medical care than men. Again, this is in agreeing with our results that some occupations found to be a significant risk factor for a subject to be a patient with chronic voice disorders as house wife's, factory workers, teachers and sale men. Moreover, low social class represented a risk factor for chronic voice disorders. Elwood et al. (1984); Hirayama (1990) and Menvielle et al. (2004) agreed that low social class found to be a significant risk factor for a subject to be a patient with chronic voice disorder. Lastly, urban residence found to be a significant risk factor for chronic voice disorders. This finding is expected and accepted in light what was previously mentioned and the relatively small number of patients from rural areas.

Regarding voice abuse (table 3), it was an important risk factor for chronic voice disorders $(\mathrm{OR}=8.33$, 95\% CI: 5.97 11.64). This result was consistent with Holinger and Johnston (1951); Cooper (1973); Kambic et al. (1981); HerringtonHall et al. (1988); Garcia et al. (1999) and Thibeault et al. (2002). Holinger and Johnston (1951) cleared that the number of women with small children, which they felt required constant disciplining was significantly high. Likewise, Cooper (1973) cleared that the homemakers were the most common group with voice disorders. Also, Herrington-Hall et al. (1988) supposed that voice abuse and the emotional conflicts that were underlie psychogenic disorders lead to a subject with voice disorders. Garcia et al. (1999) showed that voice abuse or misuse was the main risk factor in patients with chronic voice disorders. Thibeault et al. (2002) cleared that vocal overuse (excessive quantity of voice), abuse (yelling) and misuse (vocal hyperfunction with excessive muscular tension) presumably a risk factor for voice disorders. As respect smoking, it was a risk factor for voice disorders $(\mathrm{OR}=6.01$, 95\% CI: 2.29-3.95). Cigarette smoke is chronically irritating to the laryngeal mucosa and at the extreme it can provoke cancer (Hanson and Jiang, 2000). Further, Kambic et al. (1981) showed that about $50.0 \%$ of the patients with chronic voice disorders were smokers. Also, Garcia et al. (1999) found that smoking was the main risk factor for vocal folds polyps and edema, which will lead to dysphonia. Also, alcohol intake represented a risk factor for voice disorders (OR=2.90, 95\% ECL: 1.08 9.05). This result was consistent with Rothman et al. (1980); Guenel et al. (1988) and Altieri et al. (2002).

As respect results of RSI (table 4), +ve RSI was a risk factor for chronic voice disorders $\quad(\mathrm{OR}=16.94, \quad 95 \% \quad \mathrm{CI}:$ 11.8024.38). Koufman et al. (1994); El-Serag et al. (2001) and Galli et al. (2002) agreed our result. Koufman et al. (1994) found that $50.0 \%$ of their population, new patients with chronic voice disorders due to laryngeal diseases, had documented reflux disease. Reflux was also present very frequently in patients with functional dysphonia. On the other hand, reflux was infrequently found in patients with vocal fold paralysis.

The females were more commonly present in most groups of voice disorder 


\section{E. A. El-Moselhy et al}

patients; chronic laryngitis, vocal fold nodules, functional dysphonia and vocal fold polyps (table 5). While, the males were more commonly present in the miscellaneous diseases group of voice disorder patients. Previous studies of benign laryngeal lesions demonstrated a shift over the time as regard gender. Studies done before 1960 (Holinger \& Johnston, 1951 and Fitz-Hugh et al., 1958) showed about $69.0 \%$ of lesions occurred in males and $31.0 \%$ in females. Cooper (1973) reported $46.0 \%$ males and $54.0 \%$ females, which suggested a dramatic shift in the male/female percents. Also, HerringtonHall et al. (1988) found similar results when comparing benign lesions due to abuse of the voice, a significant change from $25.0 \%$ of voice disorders found in women in 1938 to $63.0 \%$ in their study. Several reasons for this increase in occurrence in women were hypothesized as increased number of women in the labor force and consequently more exposure to occupational hazards, increase numbers of cigarette smoking among them, in addition to their responsibilities as primary caretakers of the home and family. Also, some studies have shown that women appear to be more interested in health, more likely to recognize bodily changes as symptoms of an illness and more apt to seek medical care than men (Cleary, 1982 and Hibbard \& Pop, 1986). Also, functional voice disorders occurred predominantly in women (Fried, 1996). As regard age, Hanson and Jiang (2002) cleared that adults in the sixth decade of life were mainly affected with chronic laryngitis. Also, Herrington-Hall et al. (1988) detailed that chronic laryngitis occurred more commonly in the 25-44 and 45-64 year age groups, functional dysphonia in the 45-64 and 2544 year age groups and neurogenic dysphonia in the oldest age group. Coyle et al. (2001) showed that chronic laryngitis affected more commonly the 45-64 and 2544 year age groups and functional dysphonia in the 45-64 and $\geq 65$ year age groups. The patients in miscellaneous diseases group (table 5) were significantly more in the 45-46 year age group. Coyle et al. (2001) observed that vocal fold paralysis was more commonly affected the 25-44 and 45-64 year age groups, bowed vocal fold commonly affected the $\geq 65$ year age group. The 25-44 year age group was the commonest age group significantly affected with vocal fold nodules in the present study. This is consistent with Coyle et al. (2001) findings. However, Herrington-Hall et al. (1988) cleared that vocal fold nodules commonly affected the 18-24 year age group. Also, vocal fold polyps were found in the present study to occur more in the 2544 year age group. Kambic et al. (1981); Herrington-Hall et al. (1988) and Coyle et al. (2001) disagreed our result, they reported that vocal fold polyps were found to occur more in the 45-64 year age group. As respect social class, low social class was more present among the chronic laryngitis, vocal fold nodules, functional dysphonia and vocal fold polyps that caused chronic voice disorders. Elwood et al. (1984); Hirayama (1990) and Menvielle et al. (2004) agreed that low social class found to be more common among patients with chronic voice disorders. Lastly, all groups of patients with chronic voice disorders had urban residence with a statistically significant difference. Again, this finding is expected and accepted in light of what was previously mentioned and the relatively small number of patients from rural areas.

Health-related QOL represents the functional effects of an illness upon a patient as perceived by the patient. "Quality of life reflects a measure of the difference or gap between one's perceived reality and one's expectations or wishes" (Guillemin et al., 1993). Quality of life of well-being is a composite of two components; first, the ability to perform everyday activities that reflect physical, psychological and second, social well-being and patient satisfaction with levels of functioning and the control of disease and/or treatment-related symptoms (Gotay et al., 1992). The term QOL is usually used vaguely, without obvious definition (Fallowfield, 1996). This is not surprising, considering the broad nature of a concept that includes physical functioning (ability to carry out activities of daily living e.g. self care), social functioning (relationships with others and participation in social activities), psychological functioning (emotional and mental well-being) and perception of health status; pain and the overall satisfaction with life (Naughton and 
Shumaker, 1996). QOL is also a subjective concept, multidimensional scope that requires information about a range of areas of patient's life such as physical wellbeing, functional abilities, emotional and social wellbeing (De Antonio et al., 2001). In addition, it is dynamic, because it often changes across time and situations (Aronson, 1980 and Guillemin et al., 1993). Also, QOL measures subjective experiences, patient and professional can have different perspectives on what constitutes QOL. These different perspectives make it difficult to assess QOL (Gotay et al., 1992). The SF-36 broadly assesses physical, mental and social health and can be used to compare conditions and therapies (Guyatt et al., 1986 and Ware \& Sherbourne, 1992). Further, the SF-36 sensitivity to the presence of otolaryngological conditions was confirmed. Otolaryngologists are likely to use health status increasingly as health care purchasers look for greater accountability in expenditure. The SF-36 is a comprehensively validated instrument and continues to be the subject of an extensive program of ongoing research. It is easy to use, but surgeons must be able to appreciate the nuances of their findings (Benninger et al., 1998).

In this study the SF-36 questionnaire was used to assess the QOL of patients with chronic voice disorders due to laryngeal diseases and to examine the differential impact of chronic voice disorders on the various health status domains. The results of the present study confirmed that patients with chronic voice disorders due to laryngeal diseases had significantly poorer self- reported health than the controls on all the eight SF-36 subscales $(\mathrm{P}=0.000)$ (Table $6)$. These results, confirm the sensitivity of the SF-36 to the presence of oto-laryngological conditions, agreeing with the results of many studies. Smith et al. (1996) demonstrated considerable impact of dysphonia on patients' health status. Scott et al. (1997) illustrated that people with dysphonia experience social, lifestyle and employment difficulties as a direct consequence of their voice disorders. Also, Benninger et al. (1998) used the SF-36 to assess QOL in a heterogeneous group of dysphonic patients; they found significant reductions in a number of domains. Wilson et al. (2002) compared self-rated general health status as measured by the SF-36 in a large cohort of dysphonic patients with those of control group to examine the differential impact of dysphonia on the various health status domains. They concluded that dysphonic patients without obvious laryngeal disease have an adverse impact on all health status subscales as measured by the SF-36.

When comparing the SF-36 domains of the different laryngeal diseases separately that caused chronic voice disorders with control group; all domains were significantly lower in patients with chronic laryngitis, vocal fold nodules, functional dysphonia, vocal fold polyps and miscellaneous diseases group; all scored significantly lower in all domains of the SF36 than control group except bodily pain in the miscellaneous diseases group (Table 7). Benninger et al. (1998) evaluated QOL of the 3 major etiologies of dysphonia; masses, edema and vocal fold paralysis; vocal fold paralysis consistently demonstrated the worst scores of the eight SF-36 domains. Also, Spector et al. (2001) in a prospective observational outcome study of consecutive patients presenting to a laryngology clinic with unilateral vocal fold paralysis to identify the disease impact on an individual's QOL using the SF-36. They cleared that patients revealed statistically significant reductions in QOL. Carrau et al. (2004) compared patient-reported outcomes health-related QOL of the patients with laryngopharyngeal reflux with general population by the SF-36. They showed that it has a significant negative impact on the lives of patients.

\section{Conclusions And Recommendations}

It could be concluded that the most common causes of chronic voice disorders were chronic laryngitis, vocal fold nodules, functional dysphonia and vocal fold polyps. The 25-44 year age group, low social class, some jobs, urban residence and female gender were the most important sociodemographic risk factors of chronic voice disorders. Also, +ve RSI, voice abuse and smoking were important clinical risk factors. At the same time, the results of the study confirmed that patients with chronic 
voice disorders had significantly poorer self- reported health than the controls on all the eight SF-36 domains. These results emphasize the need to include a generic QOL outcome measure in the assessment of the patients with chronic voice disorders. So, it is recommended to focus research efforts, health education and clinical practices on the chronic voice disorders. More work should be conducted in more areas. Also, population based studies are needed to determine the epidemiology and QOL of the patients with chronic voice disorders in Egypt.

\section{References}

1. Abou-Seif SG, Baky FA, ElEbrashy $F$ and Gaafar HA (1991): Scleroma of the upper respiratory passages: A CT study. J Laryngol Otol., 105: 198-202.

2. Altieri A, Bosetti C, Talamini R, Gallus S, Franceschi S, Levi F, Dal-Maso L, Negri $E$ and LaVecchia C (2002): Cessation of smoking and drinking and the risk of laryngeal cancer. Br J Cancer, 18(11): 1227-9.

3. Aronson AE (1980): Clinical voice disorders: An interdisciplinary approach, New York, Marcel Dekker, 45-79.

4. Begg CB, Cho M, Eastwood S, Horton R, Moher D and Olkin I (1996): Improving the quality of reporting of randomized controlled trials. JAMA., 276: 637-9.

5. Belafsky PC, Postma GN, Amin MR and Koufman JA (2002): Symptoms and findings of laryngopharyngeal reflux. Ear Nose Throat. J., 81(2): 10-3.

6. Benninger MS, Ahuja AS and Gardner G (1998): Assessing outcomes for dysphonic patients. J Voice, 12: 540-50.

7. Bergner M, Bobbitt RA, Carter WB and Gilson BS (1981): The sickness impact profile: Development and final revision of a health status measure. Med Care, 19: 787-805.

8. Carr VI, Glaze LE, Arnold RR and Van Mersbergen $M$ (2006): Impact of voice disorders on quality of life. J Voice, 20: 17-26.
9. Carrau RL, Khidr A, Crawley JA, Hillson EM, Davis JK and Pashos CL (2004): The impact of laryngopharyngeal reflux on patient-reported quality of life. Laryngoscope, 114(4): 670-4.

10. Cleary PD, Mechanic $D$ and Greenley JR (1982): Sex differences in medical care utilization: An empirical investigation. $\mathrm{J}$ Health Social Behav., 23: 106-10.

11. Cooper M (1973): Modern techniques of vocal rehabilitation. Springfield \& Charles Thomas, 14-18.

12. Coyle SM, Weinrich BD and Stemple JC (2001): Shifts in relative prevalence of laryngeal pathology in a treatment-seeking population. J Voice, 15(3): 424-40.

13. De Antonio $M$, Davis $J$ and Nilsson C (2001): Assessment of quality of life. BMJ., 317: 1290-4.

14. El-Moselhy EA, El-Rashedy AA, El-Sawy MM, Abd El-Salam HA and Abd El-Fatah AM (2004): Laryngeal pathologies: Risk factors among adult patients seeking medical advice. Sc J Az Med Fac (Girls), 7(3): 1071-95.

15. EL-Sawy MM (2000): Conservative surgery in larynx. Thesis for MD in Otolaryngology, Faculty of Medicine, AL-Azhar University.

16. El-Serag HB, Hepworth EJ, Lee $P$ and Sonnenberg $A$ (2001): Gastroesophageal reflux disease is a risk factor for laryngeal and pharyngeal cancer. Am J Gastroenterol, 96(7): 2013-8.

17. Elwood IM, Sakamoto T, Barnes PJ and Chung KF (1984): Alcohol, smoking, social and occupational factors in the etiology of cancer of the oral cavity, pharynx and larynx. Intl Cancer, 34: 603-7.

18. Fallowfield L (1996): Quality of quality of life data. Lancet, 348: 421-6.

19. Fitz-Hugh GS, Smith DE and Chiong AT (1958): Pathology of three hundred clinically benign lesions of the vocal cords. Laryngoscope, 68: 855-9.

20. Fitzpatrick R, Davey C, Buxton $M$ and Jones DR (2001): Criteria for assessing patient based 
outcome measures for use in clinical trials. The advanced handbook of methods in evidence based health care. London, Sage, 181-94.

21. Fried MP (1996): The Larynx: A multidisciplinary approach. $2^{\text {nd }}$ ed, Chicago, Mosby-Year Book.

22. Galli J, Cammarota G, Calo L, Agostino S, D'Ugo D, Cianci R and Almadori $\mathbf{G}$ (2002): The role of acid and alkaline reflux in laryngeal squamous cell carcinoma. Laryngoscope, 112(10): 1861-5.

23. Garcia Alvarez CD, Campos Banales ME, Lopez Campos D, Rivero J, Perez Pinero $B$ and Lopez Aguado D (1999): Polyps, nodules, and Reinke's edema: An epidemiological and histopathological study. Acta Otorrinolaringol Esp, 50(6): 443-7.

24. Garratt A, Schmidt L, Mackintosh A and Fitzpatrick $\mathbf{R}$ (2002): Quality of life measurement: Bibliographic study of patient assessed health outcome measures. BMJ, 324(7351): 1417 3.

25. Gotay CC, Korn EL, McCabe MS, Moore TD and Cheson BD (1992): Building quality of life assessment into cancer treatment studies. Oncology, 6(6): 25-8.

26. Guenel P, Chastang JF, Luce D, Leclerc A and Brugere J (1988): A study of the interaction of alcohol drinking and tobacco smoking among French cases of laryngeal cancer. J Epidemiol Comm Health, 42 (4): 350-4.

27. Guillemin F, Bombardier $\mathbf{C}$ and Beaton D (1993): Cross-cultural adaptation of quality of life measures: Literature review and proposed guidelines. J Clin Epidemiol, 46: 1417-20.

28. Guyatt GH, Bombardier C and Tugwell PX (1986): Measuring disease-specific quality of life in clinical trials. Can Med Assoc J, 134: 889-95.

29. Hanson DG and Jiang JJ (2000): Diagnosis and management of chronic laryngitis associated with reflux. Am J Med, 108 (S 4a): 1125-9.

30. Hemingway H, Stafford M, Stansfield S, Shipley $M$ and Marmot M (1997): Is the
SF-36 a valid measure of change in population health? Results from the White hall II study. BMJ, 315: 1273-9.

31. Herrington-Hall $\mathbf{B}$, Lee $\mathbf{L}$, Stemple J, Niemi K and McHone M (1988): Description of laryngeal pathologies by age, gender and occupation in a treatment seeking sample. J Speech Hear Disord, 53: 57-64.

32. Hibbard JH and Pope CR (1986): Another lock at sex differences in the medical care: Illness orientation and the types of morbidities for which services are used. Women and health, 11: 2136.

33. Hirayama T (1990): Life-style and morta-lity: A large-scale census-based cohort study in Japan. In: Contributions to epidemiology and biostatistics, Wahrendorf J (Ed), Basel, Karger.

34. Holinger $\mathbf{P}$ and Johnston $\mathbf{K}$ (1951): Benign tumors of the larynx. Ann Otol Rhinol Laryngol, 60: 496-71.

35. Kambic V, Radsel Z, Zargi $M$ and Acko M (1981): Vocal cord polyps: Incidence, histology, and pathogenesis. J Laryngol Otol, 95(6): 609-18.

36. Koufman JA, Ott DJ, Ledbetter MS and Chen MY (1994): Globus pharyngeus: Radiographic evaluation and 24-hour $\mathrm{pH}$ monitoring of the pharynx and esophagus in 22 patients. Radiology, 191(1): 95-7.

37. Menvielle G, Luce D, Goldberg $P$ and Leclerc $A$ (2004): Smoking, alcohol drinking, occupational exposures and social inequalities in hypopharyngeal and laryngeal cancer. Int J Epidemiol, 11: 20-5.

38. Miller $M$ and Verdolini $K$ (1995): Frequency and risk factors for voice problems in teachers of singing and control subjects. J Voice, 9: 348- 62.

39. Naughton MJ and Shumaker SA (1996): Assessment of healthrelated quality of life. In: Fundamentals of clinical trials, Furberg CD, DeMets DL, (Eds). $3^{\text {rd }}$ ed, St Louis, Mosby Press, 32234.

40. Patrick DL and Bergner $\mathbf{M}$ (1990): Measurement of health 
status in the 1990s. Ann Rev

Public Health, 11: 165-83.

41. Rothman KJ, Cann CI, Flanders $D$ and Fried MP (1980): Epidemiology of laryngeal cancer. Epidemiology,2: 195-209.

42. Sanders C, Egger M, Donovan J, Tallon D and Frankel S (1998): Reporting on quality of life in randomized controlled trials: Bibliographic study. BMJ, 317(7167): 1191-4.

43. Scott S, Robinson $K$ and Wilson JA (1997): Patient-reported problems, risk factors for squamous epithelial carcinoma of the mouth, the oropharynx, the hypopharynx and the larynx. J Otolaryngol, 28: 78-81.

44. Smith E, Gray S, Dove H, Kirchener L and Heras $H$ (1997): Frequency and effects of teacher's voice problems. J Voice, 11: 81-7.

45. Smith E, Verdolini K and Gray S (1996): Effect of voice disorders on quality of life. J Medical Speech Lang Pathol, 4: 223-45.

46. Solomon NP, Glaze LE, Arnold RR and Van Mersbergen $M$ (2003): Effects of a vocally fatiguing task and systemic hydration on men's voices. J Voice, 17(1): 31-46.

47. Spector BC, Netterville JL, Billante C, Clary J, Reinisch $L$ and Smith TL (2001):Quality-of-life assessment in patients with unilateral vocal cord paralysis. Otolaryngol Head and Neck Surg, 125(3): 176-82.

48. Spiegel JR, Hawkshaw $M$ and Markiewicz A (2000): Acute laryngitis. Ear Nose Throat J, 79(7): 488-91.
49. Stemple J, Glaze $\mathbf{L}$ and Gerdman B (1996): Clinical voice pathology: Theory and management. $2^{\text {nd }}$ ed, San Diego, Calif, Singular Publishing Group.

50. Stewart MG, Chen AY and Stach CB (1998): Outcomes analysis of voice and quality of life in patients with laryngeal cancer. Arch Otolaryngol Head Neck Surg, 124: 143-8.

51. Thibeault SL, Gray SD and Li W (2002): Genotypic and phenotypic expressions of vocal fold polyps and Reinke's edema: A preliminary study. Ann Otol Rhinol Laryngol, 111: 302-9.

52. Thompson LD (2002):

Rhinoscleroma. Ear Nose Throat J, 81(8): 506-9.

53. Titze IR, Reinisch $L$ and Smith TL (2007): Risk factors of voice disorders. J Voice, 21: 281-7.

54. Ware JE and Sherbourne CD (1992): The MOS 36-item shortform health survey (SF-36): Conceptual framework and item selection. Med Care, 30: 473-83.

55. Ware JE, Snow KK, Kosinski M and Gandek B (1993): SF-36 health survey manual and interpretation guide. Boston: The Health Institute, New England Medical Center, 45-57.

56. Watson EK, Firman DW, Baade PD and Ring I (1996): Telephone administration of the SF-36 Health Survey: Validation studies and population norms for adults in Queensland. Aust N Z J Public Health, 20: 359-63.

57. Wilson JA, Deary IJ, Millar A and Mackenzie K (2002): The quality of life impact of dysphonia. Clin Otolaryngol Allied Scien, 27(3): 179-82. 


\section{عوامل الخطورة و نوعية الحياة \\ فى المرضي البالغين المصابين بمشاكل الصوت نوت المزياة}

عصام عبد المنعم المصيلحي - يحيا عبد العظيم بركة - إيمان شكرى عبد اللة

طارق سليمان الشوربجى **ـ محمد محمود الصاوى ***ـ طلعت محروس

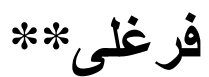

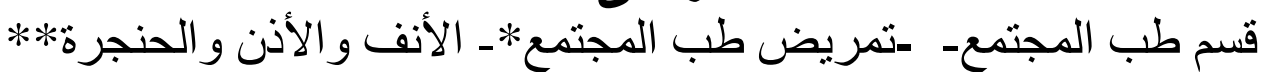

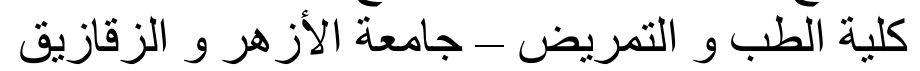

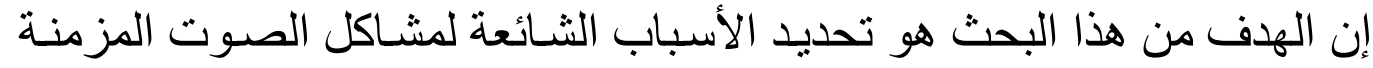

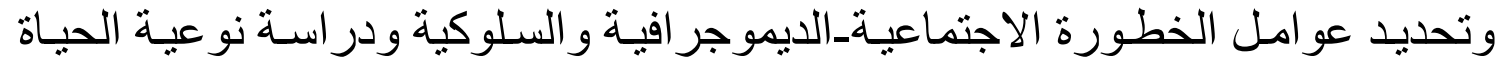

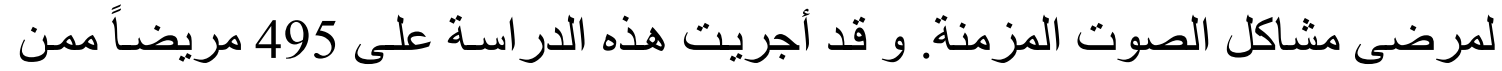

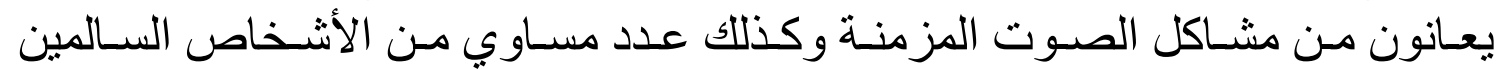

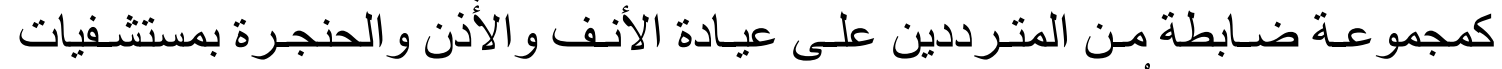

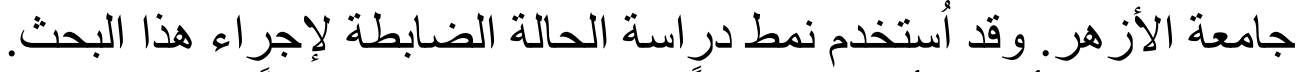

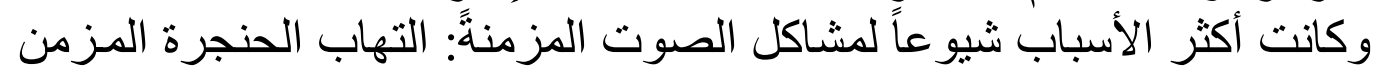

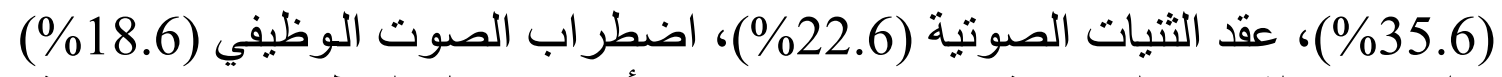

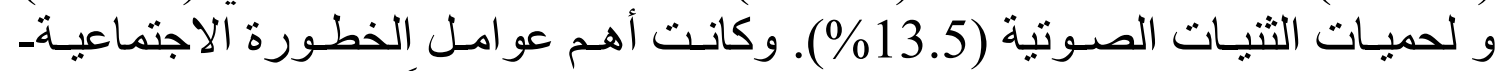

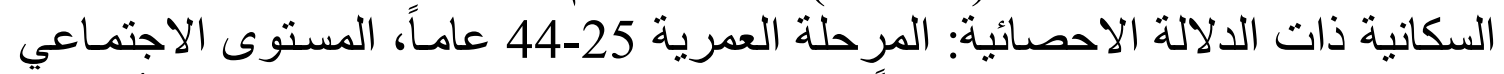
المتدنى، و كون المريض يعمل بائعاً، وسكنى المدينة، و النسـاء كجنس (نسبة أودز 


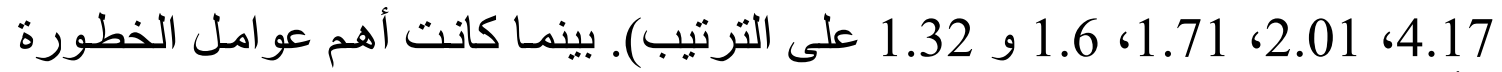

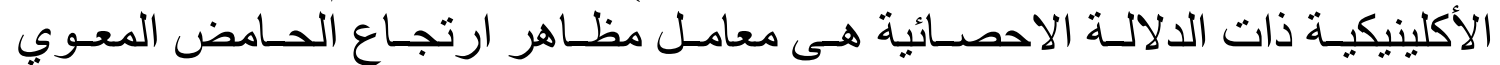

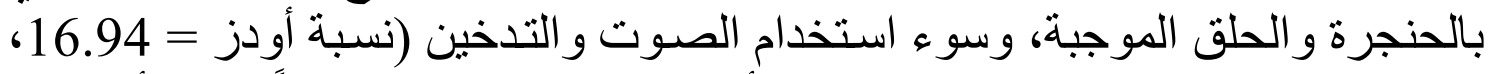

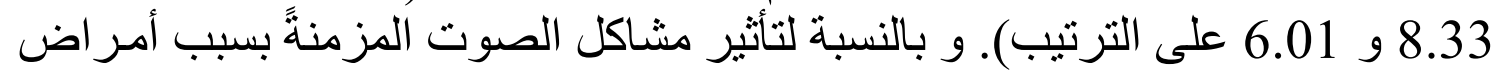

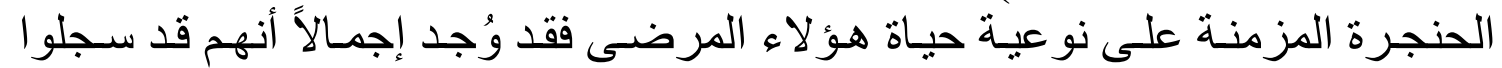

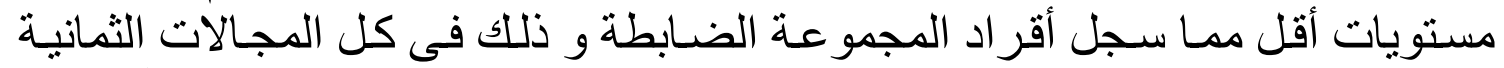

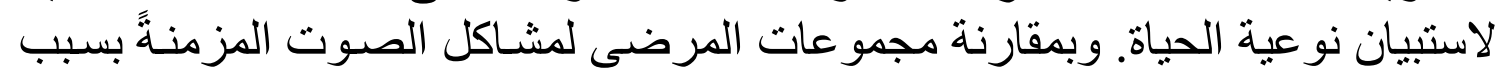

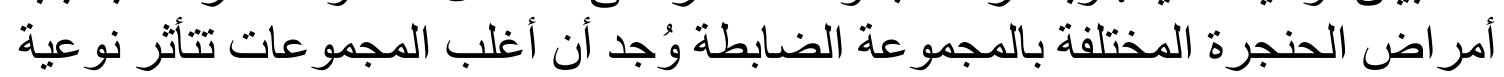

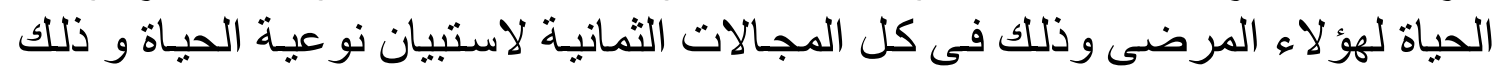

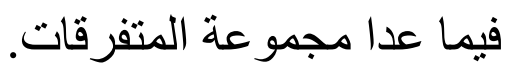

